Pacific Journal of Mathematics

ANALYTIC MAPS OF THE OPEN UNIT DISK ONTO A 


\title{
ANALYTIC MAPS OF THE OPEN UNIT DISK ONTO A GLEASON PART
}

\section{KAZUO KISHI}

\begin{abstract}
The purpose of this paper is to show that in certain uniform algebras all analytic maps (for the definition see §2) of the open unit disk onto a nontrivial Gleason part are mutually closely related (Theorem 2), and that these maps are isometries of the open unit disk with pseudo-hyperbolic metric onto a nontrivial Gleason part with part metric (Theorem 3).
\end{abstract}

The results of this paper are contained in $\S 2$. Some necessary preliminaries are given in $\S 1$.

1. Preliminaries. A uniform algebra $A$ on a compact Hausdorff space $X$ is a uniformly closed subalgebra of the algebra $C(X)$ of complex valued continuous functions on $X$ which contains the constants and separates the points of $X$. Let $\mathscr{C}(A)$ denote the maximal ideal space of $A$ which has the Gelfand topology. Let $\hat{f}$ be the Gelfand transform of $f$ in $A$ and let $\hat{A}=\{\hat{f}: f \in A\}$.

For $p$ and $\theta$ in $\mathscr{C}(A)$ we define

$$
\begin{aligned}
& G: G(\varphi, \theta)=\sup \{|\varphi(f)-\theta(f)|: f \in A,\|f\| \leqq 1\}, \\
& \sigma: \sigma(\varphi, \theta)=\sup \{|\varphi(f)|: f \in A,\|f\| \leqq 1, \theta(f)=0\},
\end{aligned}
$$

where $\|f\|=\sup \{|f(x)|: x \in X\}$, and we write $\varphi \sim \theta$ when $G(\varphi, \theta)<2$ (or, equivalently, $\sigma(\varphi, \theta)<1$ ). Then $\sim$ is an equivalence relation in $\mathscr{C}(A)$, and an equivalence class $P(m)=\{\varphi: \varphi \in \mathscr{C}(A), \varphi \sim m\}(\supsetneqq\{m\})$ is called the (nontrivial) Gleason part of $m \in \mathscr{M}(A)$ (cf. Gleason [2]). $G(\varphi, \theta)$ and $\sigma(\varphi, \theta)$ are metrics on $P(m)$ (cf. König [5]).

If $m \in \mathscr{L}(A)$ has a unique representing measure $\mu_{m}$, i.e., if $m$ has a unique probability measure $\mu_{m}$ on $X$ such that $m(f)=\int f d \mu_{m}$ for every $f \in A$, then every $\varphi$ in $P(m)$ also has a unique representing measure $\mu_{\varphi}$. It is also known that $\varphi$ in $M_{A}$ belongs to $P(m)$ if and only if there exist mutually absolutely continuous representing measures $\mu_{\varphi}, \mu_{m}$ for $\varphi, m$ respectively; indeed, there exists a constant $c(0<c<1)$ such that $c \mu_{\varphi} \leqq \mu_{m}$ and $c \mu_{m} \leqq \mu_{\varphi}$.

For example, let $A(D)$ denote the disk algebra of all continuous functions on the closed unit disk $\bar{D}=\{s:|s| \leqq 1\}$ in the plane wich are analytic in the open unit disk $D$. Then $\mathscr{C}(A(D))$ can be identified with $\bar{D}$, and the open unit disk $D$ is one part. For $t, s \in D$, we see that 


$$
\begin{aligned}
G(t, s) & =\sup \{|f(t)-f(s)|: f \in A(D),\|f\| \leqq 1\} \\
\sigma(t, s) & =\sup \{|f(t)|: f \in A(D),\|f\| \leqq 1, f(s)=0\} \\
& =\left|\frac{t-s}{1-\bar{s} t}\right| \quad \text { (pseudo-hyperbolic metric) } .
\end{aligned}
$$

Throughout the rest of this paper, we do not distinguish in notations $\phi \in \mathscr{C}(A)$ from its representing measure when $\phi$ has a unique representing measure, and we suppose that $m \in \mathscr{M}(A)$ has a unique representing measure $m$. Let $A_{m}=\{f \in A ; m(f)=0\}$, the kernel of a complex homomorphism $m$. Let $H^{\infty}(m), H_{m}^{\infty}$ be the weakstar closures of $A, A_{m}$ in $L^{\infty}(m)$ respectively, and for $1 \leqq p<\infty$ let $H^{P}(m), H_{m}^{P}$ be the closures of $A, A_{m}$ in $L^{P}(m)$ norm respectively. If $\varphi$ belongs to $P(m)(\supsetneqq\{m\})$, then for $1 \leqq p<\infty$ the spaces $H^{P}(m)$ and $H^{P}(\varphi)$ are identical as sets of (equivalence classes of) functions; as Banach spaces, they have distinct but equivalent norms. Under the same hypothesis, the Banach algebras $H^{\infty}(m)$ and $H^{\infty}(\varphi)$ are identical.

For a Dirichlet algebra Wermer [7] showed the following theorem, and Hoffman [3] generalized Wermer's result to a logmodular algebra (cf. Browder [1], Chap. IV). Functions in $H^{\infty}(m)$ of unit modulus are called inner functions.

Theorem 1 (Wermer's Embedding Theorem). Let $A$ be a uniform algebra on a compact space $X$. Suppose that $m \in \mathscr{M}(A)$ has a unique representing measure $m$ on $X$, and that the part $P$ of $m$ consists of more than one point. Then we have the following.

(1) There is an inner function $Z(=$ Wermer's embedding function) such that $Z H^{2}(m)=H_{m}^{2}$.

(2) If $\varphi \in P$, define $\hat{Z}(\varphi)=\int Z d \varphi$. Then $\hat{Z}$ is a one-one map of the part $P$ onto the open unit disk $D$ in the plane. The inverse map $\tau$ of $\hat{Z}$ is a one-one continuous map of $D$ onto $P$ (with the Gelfand topology).

(3) For every $f$ in $A$ the composed function $\hat{f}_{\circ} \tau$ is analytic on $D$.

Let $\varphi$ be an element of the Gleason part $P(m)$ of $m$ in $\mathscr{C}(A)$. Then there is a function $h$ in $L^{\infty}(m)$ such that $\varphi(f)=\int f d \varphi=\int f h d m$ for all $f \in A$, so $\varnothing$ has a unique extension to a linear functional $\widetilde{\phi}$ on $H^{\infty}(m)$ which is both multiplicative and weak-star continuous. For any $f \in H^{\infty}(m) \tilde{\varphi}$ has the form

$$
\tilde{\varphi}(f)=\int f d \varphi=\int f h d m
$$


We call $\tilde{\varphi}$ the measure extension of $\varphi$ in $P(m)$.

Proposition. Let $A, m, P(m)$ and $Z$ be as in Wermer's embedding theorem. Let $\mathscr{P}=\mathscr{P}(m)$ be the set of measure extension $\tilde{\varphi}$ of $\varphi$ in $P(m)$. Then we have the following.

(1) $\mathscr{P}$ is the nontrivial Gleason part of $\tilde{m}$ in $\mathscr{C}\left(H^{\infty}(m)\right)$.

(2) The map $\hat{Z} \mid \mathscr{P}^{\text {is a a }}$ one-one continuous map of the part $\mathscr{P}$ (with the Gelfand topology) onto the open unit disk $D$, and thus the inverse map $\tilde{\tau}$ of $\hat{Z} \mid \mathscr{P}$ is a homeomorphism of $D$ onto $\mathscr{P}$.

Proof. If the Gelfand transform $\hat{H}^{\infty}(m)=\hat{H}^{\infty}$ of $H^{\infty}(m)$ is restricted to the maximal ideal space $Y$ of $L^{\infty}(m)$, then $\hat{H}^{\infty}$ is a logmodular algebra on $Y$ (see Hoffman [3], Theorem 6.4, corollary), and therefore every complex homomorphism $\varphi$ of $H^{\infty}(m)$ has a unique representing measure on $Y$ (see [3], Theorem 4.2). In particular, $\tilde{m} \in \mathscr{M}\left(H^{\infty}(m)\right)$ has a unique representing (normal) measure $\tilde{m}$ on the hyperstonean space $Y$. Then for every $f$ in $L^{\infty}(m)$ we have

$$
\int_{X} f d m=\int_{Y} \hat{f} d \tilde{m}
$$

And we can identify $L^{\infty}(\tilde{m})$ with $C(Y)=\hat{L}^{\infty}(m)$ (cf. Srinivasan-Wang [6], pp. 221-223).

Now let $\Sigma$ be the Gleason part of $\widetilde{m}$. For $\widetilde{\varphi}$ in $\mathscr{P}$, we have

$$
\widetilde{\phi}(f)=\int_{X} f d \varphi=\int_{X} f h d m=\int_{Y} \hat{f} \hat{h} d \tilde{m}\left(f \in H^{\infty}(m)\right),
$$

where $h$ is a function in $L^{\infty}(m)$ with $a<h<b$ for some positive constants $a$ and $b$. From this we see that $\widetilde{\varphi}$ is in $\Sigma$.

Conversely if $\lambda$ is an element of $\Sigma$, then $\lambda$ has a unique representing measure $\tilde{\lambda}$ on $Y$, and we have for every $f \in H^{\infty}(m)$

$$
\lambda(f)=\int_{Y} \hat{f} d \tilde{\lambda}=\int_{Y} \hat{f} \frac{d \tilde{\lambda}}{d \tilde{m}} d \tilde{m} .
$$

Since $d \tilde{\lambda} / d \widetilde{m}$ is an element of $L^{\infty}(\widetilde{m})$, there is a function $h$ in $L^{\infty}(m)$ such that $d \tilde{\lambda} / d \tilde{m}=\hat{h}$ a.e. $(d \tilde{m})$. Hence we have

$$
\lambda(f)=\int_{Y} \hat{f} \hat{h} d \tilde{m}=\int_{X} f h d m
$$

and thus $\lambda \in \mathscr{P}$. So we get $\mathscr{P}=\Sigma$. Then the rest part (2) of the proposition follows from Theorem 1.

2. Results.

Definition. Let $P(m)$ be the nontrivial Gleason part of $m$ in 
the maximal ideal space $\mathscr{C}(A)$ of a uniform algebra $A$. A one-one continuous map $\rho(t)$ of the open unit disk $D$ onto $P(m)$ (with the Gelfand topolgy) is called an analytic map if the composition $\hat{f}(\rho(t))$ is analytic on $D$, for every $f$ in $A$.

Now we are in a position to prove the following theorem.

THeOREM 2. Let $A$ be a uniform algebra on a compact space $X$. Suppose that $m \in \mathscr{M}(A)$ has a unique representing measure $m$ on $X$, and that the part $P$ of $m$ consists of more than one point. Let $\tau(t)$ be an analytic map of the open unit disk $D$ onto $P$ which is obtained in Theorem 1. If $\rho(t)$ is an analytic map of $D$ onto $P$ such that $\rho(\alpha)=m$, then we have

$$
\rho(t)=\tau\left(\beta \frac{t-\alpha}{1-\bar{\alpha} t}\right)
$$

where $\beta$ is a constant of modulus 1. Furthermore, $\tau(t)$ is a homeomorphism if and only if $\rho(t)$ is a homeomorphism.

Proof. Let $\mathscr{P}, Z$, and $\tilde{\tau}$ be as in Theorem 1 and proposition. For any $t \in D, \rho(t)$ has a unique representing measure $h_{t} d m$, where $h_{t}$ is an element of $L^{\infty}(m)$. Let $\tilde{\rho}(t)$ be the measure extension of $\rho(t)$ i.e., $\tilde{\rho}(t)(f)=\int f h_{t} d m$ for all $f \in H^{\infty}(m)$. For each $f \in H^{\infty}(m)$ there exists a sequence $\left\{f_{n}\right\}$ in $A$ such that $\left\|f_{n}\right\| \leqq\|f\|$ for all $n$ and $f_{n} \rightarrow f$ a.e. $(d m)$ (Hoffman-Wermer theorem, see [1], Theorem 4.2.5). Then, by Lebesgue's dominant convergence theorem, $\rho(t)\left(f_{n}\right)=\int f_{n} h_{t} d m \rightarrow \tilde{\rho}(t)(f)$ for every $t$ in $D$. Since $\rho(t)\left(f_{n}\right)(n=1,2, \cdots)$ are analytic in $D$ and $\left|\rho(t)\left(f_{n}\right)\right| \leqq\left\|f_{n}\right\| \leqq\|f\|$, we see that, for every $f$ in $H^{\infty}(m), \tilde{\rho}(t)(f)$ is analytic in $D$ (Vitali's theorem). Hence we see that $\tilde{\rho}(t)$ is an analytic map of $D$ onto $\mathscr{P}$. If we set $g(t)=\left(\tilde{\tau}^{-1} \circ \tilde{\rho}\right)(t)=\hat{Z}(\tilde{\rho}(t))$, then $f(t)$ is a one-one holomorphic map of $D$ onto $D$, and $g(\alpha)=0$. Hence we see

$$
g(t)=\beta \frac{t-\alpha}{1-\bar{\alpha} t},
$$

where $\beta$ is a constant of modulus 1 , and thus we have

$$
\tilde{\tau}\left(\beta \frac{t-\alpha}{1-\bar{\alpha} t}\right)=\tilde{\rho}(t)
$$

Since $\tilde{\tau}(t) \mid A=\tau(t)$ and $\tilde{\rho}(t) \mid A=\rho(t)$ we have

$$
\tau\left(\beta \frac{t-\alpha}{1-\bar{\alpha} t}\right)=\rho(t) .
$$


Next we prove that $\tau(t)$ is a homeomorphism if and only if $\rho(t)=$ $\tau(\beta(t-\alpha) /(1-\bar{\alpha} t))$ is a homeomorphism. We put $L_{\alpha}(t)=(t+\alpha) /(1+\bar{\alpha} t)$ and $\beta=e^{i \theta}$. Then $\tau(t)$ is a homeomorphism of $D$ onto $P$ if and only if $\hat{Z}(\varphi)\left(=\int Z d \varphi\right)$ is a continuous map of $P$ onto $D$ if and only if $L_{\alpha} \circ e^{-\imath \theta_{\circ}} \hat{Z}$ is a continuous map of $P$ onto $D$ if and only if

$$
\left(L_{\alpha} \circ e^{-\imath \theta} \circ \hat{Z}\right)^{-1}(t)=\tau\left(e^{i \theta} L_{-\alpha}(t)\right)=\tau\left(e^{i \theta} \frac{t-\alpha}{1-\bar{\alpha} t}\right)=\rho(t)
$$

is a homeomorphism, and the theorem is proved.

Next we shall prove the following theorem which generalizes a formula (6.12) in Hoffman [4], p. 105.

Theorem 3. Let $A$ be a uniform algebra on $X$. Suppose that $m \in \mathscr{C}(A)$ has a unique representing measure $m$ on $X$, and that the part $P$ of $m$ consists of more than one point. If $\rho(t)$ is an analytic map of the open unit disk $D$ onto $P(m)$, then we have

$$
\begin{aligned}
\sigma(\rho(t), \rho(s)) & =\sigma(t, s), \\
G(\rho(t), \rho(s)) & =G(t, s) .
\end{aligned}
$$

For the definitions of $\sigma, G$ see $(1.1) \sim(1.4)$.

Proof. Let $Z, \mathscr{P}, \tau$ and $\tilde{\tau}$ be as Theorem 1 and proposition. Let $\tilde{\tau}(t)=\widetilde{\varphi}, \tilde{\tau}(s)=\widetilde{\theta}, \tau(t)=\varphi$ and $\tau(s)=\theta$. From Lemma 4.4 .4 in Browder [1], we see that

$$
f \in H_{\widetilde{\theta}}^{\infty}=\left\{f: f \in H^{\infty}(m)=H^{\infty}(\theta), \tilde{\theta}(f)=\int f d \theta=0\right\}
$$

if and only if $f \in(Z-s) H^{\circ}(m)$, and from this we easily get $H_{\tilde{\theta}}^{\infty}=$ $\{(Z-s) /(1-\bar{s} Z)\} H^{\infty}(m)$. So we have

$$
\begin{aligned}
& \sigma(\widetilde{\varphi}, \widetilde{\theta})=\sup \left\{|\widetilde{\varphi}(f)| ; f \in H^{\infty}(m),\|f\| \leqq 1, \widetilde{\theta}(f)=0\right\} \\
& =\sup \left\{|\widetilde{\varphi}(f)|: f \in \frac{Z-s}{1-\bar{s} Z} H^{\infty}(m),\|f\| \leqq 1\right\} \\
& =\sup \left\{\left|\widetilde{\varphi}\left(\frac{Z-s}{1-\bar{s} Z}\right) \widetilde{\rho}(g)\right|: g \in H^{\infty}(m),\|g\| \leqq 1\right\} \\
& =\left|\frac{t-s}{1-\bar{s} t}\right|=\sigma(t, s) .
\end{aligned}
$$

Since the closures of $A_{\theta}$ and $H_{\widetilde{\theta}}^{\infty}$ in $L^{2}(m)$ are the same $H_{\theta}^{2}=\{f: f \in$ $\left.H^{2}(m)=H^{2}(\theta), \int f d \theta=0\right\}$, we have the following equalities from the result which is stated as "the perhaps surprising equality" in 
Browder [1], p. 134. (Note that $\widetilde{\varphi}(f)=\int_{Y} \hat{f} \hat{h} d \tilde{m}=\int_{Y} \hat{f} d \tilde{\varphi}$ (see (1.5)) and $\int_{X}|f|^{2} d \varphi=\int_{Y}|\hat{f}|^{2} d \widetilde{\varphi}$, for any $f \in H_{\theta}^{\infty}$.)

$$
\begin{aligned}
\sigma(\varphi, \theta) & =\sup \left\{|\varphi(f)|: f \in A_{\theta},\|f\| \leqq 1\right\} \\
& =\sup \left\{|\varphi(f)|: f \in A_{\theta}, \int|f|^{2} d \varphi \leqq 1\right\} \\
& =\sup \left\{|\varphi(f)|: f \in H_{\theta}^{2}, \int|f|^{2} d \varphi \leqq 1\right\} \\
& =\sup \left\{|\widetilde{\varphi}(f)|: f \in H_{\tilde{\theta}}^{\infty}, \int|f|^{2} d \varphi \leqq 1\right\} \\
& =\sup \left\{|\widetilde{\varphi}(f)|: f \in H_{\tilde{\theta}}^{\infty},\|f\| \leqq 1\right\} \\
& =\sigma(\widetilde{\varphi}, \tilde{\theta}) .
\end{aligned}
$$

Hence we have

$$
\sigma(\tau(t), \tau(s))=\sigma(\widetilde{\tau}(t), \widetilde{\tau}(s))=\sigma(t, s) .
$$

If $\rho(t)$ is an analytic map of $D$ onto $P(m)$, then by Theorem 2 we have $\rho(t)=\tau(\beta(t-\alpha) /(1-\bar{\alpha} t))$, where $\beta$ is a constant of modulus 1 . Therefore we have

$$
\sigma(\rho(t), \rho(s))=\sigma\left(\beta \frac{t-\alpha}{1-\bar{\alpha} t}, \beta \frac{s-\alpha}{1-\bar{\alpha}_{s}}\right)=\sigma(t, s) .
$$

The following equality is proved by König [5], which holds for $\varphi, \theta$ in the maximal ideal space $\mathscr{C}(A)$ of any uniform algebra $A$.

$$
2 \log \frac{2+G(\varphi, \theta)}{2-G(\varphi, \theta)}=\log \frac{1+\sigma(\varphi, \theta)}{1-\sigma(\varphi, \theta)} \text {. }
$$

Using this we get

$$
G(\rho(t), \rho(s))=G(t, s) \text {. }
$$

\section{REFERENCES}

1. A. Browder, Introduction to Function Algebras, W. A. Benjamin, New York, 1969. 2. A. Gleason, Function Algebras, Seminar on analytic functions, Vol. II, Institute for Advanced Study, Princeton, 1957, 217-226.

3. K. Hoffman, Analytic functions and logmodular Banach algebras, Acta Math., 108 (1962), 271-317.

4. — Bounded analytic functions and Gleason parts, Ann. of Math., 86 (1967), $74-111$.

5. H. König, On the Gleason and Harnack metrics for uniform algebras, Proc. Amer. Math. Soc., 22 (1969), 100-101.

6. T. P. Srinivasan and Ju-kwei Wang, Weak*-Dirichlet Algebras, Proc. Internat. Sympos. on Function Algebras (Tulane Univ.), Scott, Foresman, Chicago, (1965), 216-249.

7. J. Wermer, Dirichlet algebras, Duke Math. J., 27 (1960), 273-282.

Received July 20, 1973.

Wakayama University, Wakayama, Japan 


\title{
PACIFIC JOURNAL OF MATHEMATICS
}

\author{
EDITORS
}

\author{
RICHARD ARENS (Managing Editor) \\ University of California \\ Los Angeles, California 90024
}

\author{
R. A. Beaumont \\ University of Washington \\ Seattle, Washington 98105
}

J. DugundjI

Department of Mathematics

University of Southern California

Los Angeles, California 90007

D. Gilbarg and J. Milgram

Stanford University

Stanford, California 94305

\section{ASSOCIATE EDITORS}
E. F. BECKENBACH
B. H. NEUMANN
F. WOLF
K. Yoshida

\section{SUPPORTING INSTITUTIONS}

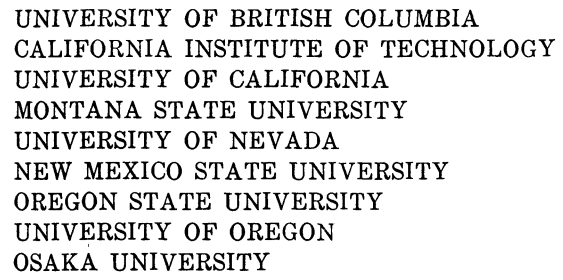

UNIVERSITY OF BRITISH COLUMBIA CALIFORNIA INSTITUTE OF TECHNOLOGY UNIVERSITY OF CALIFORNIA MONTANA STATE UNIVERSITY UNIVERSITY OF NEVADA NEW MEXICO STATE UNIVERSITY OREGON STATE UNIVERSITY UNIVERSITY OF OREGON OSAKA UNIVERSITY

\author{
UNIVERSITY OF SOUTHERN CALIFORNIA \\ STANFORD UNIVERSITY \\ UNIVERSITY OF HAWAII \\ UNIVERSITY OF TOKYO \\ UNIVERSITY OF UTAH \\ WASHINGTON STATE UNIVERSITY \\ UNIVERSITY OF WASHINGTON

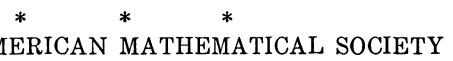

The Supporting Institutions listed above contribute to the cost of publication of this Journal, but they are not owners or publishers and have no responsibility for its content or policies.

Mathematical papers intended for publication in the Pacific Journal of Mathematics should be in typed form or offset-reproduced, (not dittoed), double spaced with large margins. Please do not use built up fractions in the text of your manuscript. You may however, use them in the displayed equations. Underline Greek letters in red, German in green, and script in blue. The first paragraph or two must be capable of being used separately as a synopsis of the entire paper. Items of the bibliography should not be cited there unless absolutely necessary, in which case they must be identified by author and Journal, rather than by item number. Manuscripts, in triplicate, may be sent to any one of the editors. Please classify according to the scheme of Math. Reviews, Index to Vol. 39. All other communications should be addressed to the managing editor, or Elaine Barth, University of California, Los Angeles, California, 90024.

The Pacific Journal of Mathematics expects the author's institution to pay page charges, and reserves the right to delay publication for nonpayment of charges in case of financial emergency.

100 reprints are provided free for each article, only if page charges have been substantially paid. Additional copies may be obtained at cost in multiples of 50 .

The Pacific Journal of Mathematics is issued monthly as of January 1966. Regular subscription rate: $\$ 72.00$ a year (6 Vols., 12 issues). Special rate: $\$ 36.00$ a year to individual members of supporting institutions.

Subscriptions, orders for back numbers, and changes of address should be sent to Pacific Journal of Mathematics, 103 Highland Boulevard, Berkeley, California, 94708.

PUBLISHED BY PACIFIC JOURNAL OF MATHEMATICS, A NON-PROFIT CORPORATION

Printed at Kokusai Bunken Insatsusha (International Academic Printing Co., Ltd.), 8-8, 3-chome, Takadanobaba, Shinjuku-ku, Tokyo 160, Japan.

Copyright (C) 1975 by Pacific Journal of Mathematics Manufactured and first issued in Japan 


\section{Pacific Journal of Mathematics}

\section{Vol. 63, No. 2 \\ April, 1976}

Joseph Anthony Ball and Arthur R. Lubin, On a class of contractive perturbations

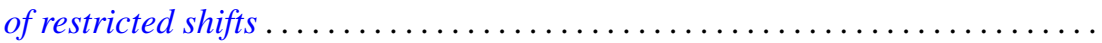

Joseph Becker and William C. Brown, On extending higher derivations generated

by cup products to the integral closure .......................

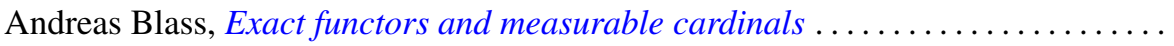

Joseph Eugene Collison, A variance property for arithmetic functions . . . . . . . . . .

Craig McCormack Cordes, Quadratic forms over nonformally real fields with a

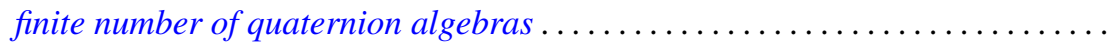

Freddy Delbaen, Weakly compact sets in $H^{1} \ldots \ldots \ldots \ldots \ldots \ldots \ldots \ldots \ldots$

G. D. Dikshit, Absolute Nörlund summability factors for Fourier series ..........

Edward Richard Fadell, Nielsen numbers as a homotopy type invariant. . ........

Josip Globevnik, Analytic extensions of vector-valued functions . . . . . . . . . . . .

Robert Gold, Genera in normal extensions . . . . . . . . . . . . . . . . . . . 389

Solomon Wolf Golomb, Formulas for the next prime

Robert L. Griess, Jr., The splitting of extensions of $S L(3,3)$ by the vector space

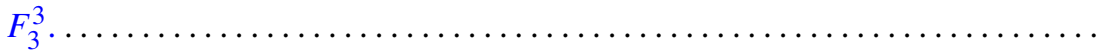

Thomas Alan Keagy, Matrix transformations and absolute summability .........

Kazuo Kishi, Analytic maps of the open unit disk onto a Gleason part.

Kwangil Koh, Jiang Luh and Mohan S. Putcha, On the associativity and commutativity of algebras over commutative rings . ..... . .

James C. Lillo, Asymptotic behavior of solutions of retarded differential difference equations.

John Alan MacBain, Local and global bifurcation from normal eigenvalues ..

Anna Maria Mantero, Sets of uniqueness and multiplicity for $L^{p}$

J. F. McClendon, Embedding metric families

L. Robbiano and Giuseppe Valla, Primary powers of a prime ideal .

Wolfgang Ruess, Generalized inductive limit topologies and barrelledness

properties.

Judith D. Sally, Bounds for numbers of generators of Cohen-Macaulay ideals

Helga Schirmer, Mappings of polyhedra with prescribed fixed points and fixed point indices.

Cho Wei Sit, Quotients of complete multipartite graphs

S. Sznajder and Zbigniew Zielezny, Solvability of convolution equations in $\mathscr{K}_{p}^{\prime}$,

$p>1$.

Mitchell Herbert Taibleson, The existence of natural field structures for finite

dimensional vector spaces over local fields

William Yslas Vélez, A characterization of completely regular fields

P. S. Venkatesan, On right unipotent semigroups ..............

Kenneth S. Williams, A rational octic reciprocity law ............

Robert Ross Wilson, Lattice orderings on the real field .......... 